\title{
Long Cycles in Graphs with Large Degree Sums and Neighborhood Unions
}

J. van den Heuvel* FACULTY OF APPLIED MATHEMATICS, UNIVERSITY OF TWENTE P.O. BOX 217, 7500 AE ENSCHEDE, THE NETHERLANDS

\section{ABSTRACT}

We present and prove several results concerning the length of longest cycles in 2 connected or 1-tough graphs with large degree sums. These results improve many known results on long cycles in these graphs. We also consider the sharpness of the results and discuss some possible strengthenings. (c) 1996 John Wiley \& Sons, Inc.

\section{INTRODUCTION AND RESULTS}

We use Bondy and Murty [5] for terminology and notation not defined here and consider simple graphs only. If $G$ is a graph and $S \subseteq V(G)$, then $N(S)$ denotes the neighborhood of $S$, that is, the set of all vertices in $G$ adjacent to at least one vertex in $S$. If $S=\{u\}$, we write $N(u)$ instead of $N(\{u\})$ and set $d(u)=|N(u)|$. We write $\omega(G)$ for the number of components of $G$ and $\alpha(G)$ for its vertex independence number.

For an integer $t \geq 1$ we define the parameters $\sigma_{t}(G)$ and $N C_{t}(G)$ by

$$
\begin{aligned}
\sigma_{t}(G) & =\min \left\{\sum_{v \in S} d(v) \mid S \subseteq V(G) \text { is an independent set with }|S|=t\right\}, \\
N C_{t}(G) & =\min \{|N(S)| \mid S \subseteq V(G) \text { is an independent set with }|S|=t\} .
\end{aligned}
$$

In these definitions we follow the convention that the minimum over an empty set is $+\infty$. The definitions imply that $\sigma_{1}(G)=\mathrm{NC}_{1}(G)=\delta(G)$ (the minimum vertex degree of $G$ ), and

\footnotetext{
*Current address: Department of Mathematics and Statistics, Simon Fraser University, Burnaby, B.C., Canada V5A 1 S6.
} 
$\mathrm{NC}_{t} \geq(1 / t) \sigma_{t}(G) \geq \delta(G)$ in general. We define $\bar{\delta}(G)=\left\lceil\frac{1}{3} \sigma_{3}(G)\right\rceil$, so that

$$
\mathrm{NC}_{3}(G) \geq \bar{\delta}(G) \geq \frac{1}{3} \sigma_{3}(G) \geq \delta(G)
$$

A graph $G$ is called 1 -tough if $\omega(G-S) \leq|S|$ for every nonempty subset $S$ of $V(G)$. Let $G_{1}(n)$ denote the class of 1-tough graphs on $n \geq 3$ vertices such that $\sigma_{3}(G) \geq n$, and let $G_{2}(n)$ denote the class of 2 -connected graphs on $n$ vertices such that $\sigma_{3}(G) \geq n+2$.

The lengths of a longest path and a longest cycle in $G$ (the numbers of edges in a longest path and a longest cycle) are denoted by $p(G)$ and $c(G)$, respectively.

A classical theorem from Dirac [7] states that a graph $G$ on $n \geq 3$ vertices with $\delta(G) \geq \frac{1}{2} n$ contains a Hamilton cycle. This result was generalized as follows.

Theorem 1 (Nash-Williams [13]). If $G$ is a 2-connected graph on $n$ vertices such that $\delta(G) \geq \max \left\{\frac{1}{3}(n+2), \alpha(G)\right\}$, then $G$ contains a Hamilton cycle.

By (1), Theorem 1 is implied by Theorem 2.

Theorem 2 (Bauer, Morgana, Schmeichel and Veldman [2]). If $G \in \mathcal{G}_{2}(n) \cup G_{1}(n)$, then $c(G) \geq \min \left\{n, n+\frac{1}{3} \sigma_{3}(G)-\alpha(G)\right\}$.

Theorem 2 implies several known results, as shown in the surveys Bauer, Broersma and Veldman [1] and Bauer, Schmeichel and Veldman [3]. Here we further generalize Theorem 2.

\section{Theorem 3.}

(a) If $G \in G_{2}(n)$ and $t \in \mathbb{N}$, then

$$
c(G) \geq \min \left\{n, \frac{1}{2}(n+3 \bar{\delta}(G)+1-t), n+\mathrm{NC}_{1}(G)-\alpha(G)\right\} .
$$

(b) If $G \in G_{1}(n)$ and $t \in \mathbb{N}$, then

$$
c(G) \geq \min \left\{n, \frac{1}{2}(n+3 \bar{\delta}(G)+4-t), n+\mathrm{NC}_{f}(G)-\alpha(G)\right\}
$$

Theorem 3 implies Theorem 2 by (1). The proof of Theorem 3 is postponed to Section 5 .

Theorem 3 would not be true if the lower bounds on $\sigma_{3}(G)$ were relaxed in the definitions of $G_{2}(n)$ and $G_{1}(n)$; this is already the case for Theorem 2 as is shown by examples in [2]. Here we present examples showing that both the lower bounds on $c(G)$ and the subscripts of $\mathrm{NC}(G)$ in the conclusion of Theorem 3 (a) cannot be increased in general.

Let $k, \ell, m \in \mathbb{N}$ such that $2 k \geq \ell+2 m+2$ and $\ell+m \geq k+1$ (hence $k \geq m+3$ ). Define the graph $G_{k, \ell, m}$ as the join of $K_{k}$ and $\ell K_{1}+m K_{2}$, where "+" denotes the union of graphs (see Fig. 1). $G_{k, \ell, m}$ is a 2-connected graph with $n=\left|V\left(G_{k, \ell, m}\right)\right|=k+\ell+2 m$, $\sigma_{3}\left(G_{k, \ell, m}\right)=3 k \geq n+2, \alpha\left(G_{k, \ell, m}\right)=\ell+m$ and $c\left(G_{k, \ell, m}\right)=2 k+m$. Furthermore, we have $\bar{\delta}\left(G_{k, \ell, m}\right)=k$ and $\mathrm{NC}_{\ell+x}\left(G_{k, \ell, m}\right)=k+x$ if $0 \leq x \leq m$. This means that for $t=\ell$ we have

$$
\begin{aligned}
\frac{1}{2}\left(n+3 \bar{\delta}\left(G_{k, \ell, m}\right)+1-t\right) & =2 k+m+\frac{1}{2}>c\left(G_{k, \ell, m}\right), \\
n+\mathrm{NC}_{t}\left(G_{k, \ell, m}\right)-\alpha\left(G_{k, \ell, m}\right) & =2 k+m=c\left(G_{k, \ell, m}\right),
\end{aligned}
$$




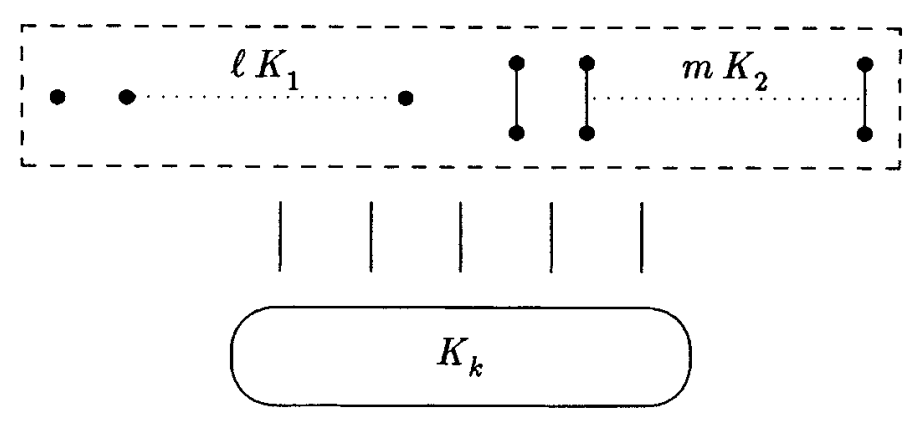

FIGURE 1. The graph $G_{k, \ell, m}$. The vertical lines indicate a join.

and for $t=\ell+1$ we obtain

$$
\begin{aligned}
\frac{1}{2}\left(n+3 \bar{\delta}\left(G_{k, \ell, m}\right)+1-t\right) & =2 k+m=c\left(G_{k, \ell, m}\right), \\
n+\mathrm{NC}_{t}\left(G_{k, \ell, m}\right)-\alpha\left(G_{k, \ell, m}\right) & =2 k+m+1>c\left(G_{k, \ell, m}\right) .
\end{aligned}
$$

Hence Theorem 3(a) is best possible if $\sigma_{3}(G) \equiv 0(\bmod 3)$. The graphs obtained from $G_{k, \ell, m}$ by deleting one or two edges incident with an isolated vertex of $G_{k, \ell, m}-V\left(K_{k}\right)$ show that Theorem $3\left(\right.$ a) is also best possible if $\sigma_{3}(G) \not \equiv 0(\bmod 3)$.

Note that the graphs $G_{k, \ell, m}$ satisfy $\left|V\left(G_{k, \ell, m}\right)\right|-c\left(G_{k, \ell, m}\right)=\ell+m-k$. The graphs $H_{k, \ell, 1}$ to be introduced in Section 2 can be used to show that Theorem 3(b) is sharp for graphs $G$ in $G_{1}(n)$ with $|V(G)|-c(G)=1$. We do not know of the existence of graphs $G \in G_{1}(n)$ with $|V(G)|-c(G) \geq 2$ for which Theorem $3(\mathrm{~b})$ is best possible. This is why we believe an improvement of Theorem 3(b) is possible. We discuss this further in Section 2.

Theorem 3 generalizes several known results. A first corollary is the following.

Corollary 4 (Broersma, Van den Heuvel, and Veldman [6]).

(a) If $G \in G_{2}(n)$, then $c(G) \geq \min \left\{n, n+\mathrm{NC}_{3} \bar{\delta}(G)-n+2(G)-\alpha(G)\right\}$.

(b) If $G \in G_{1}(n)$, then $c(G) \geq \min \left\{n, n+\mathrm{NC}_{3} \bar{\delta}(G)-n+5(G)-\alpha(G)\right\}$.

Proof (a) Set $t=3 \bar{\delta}(G)-n+2$, hence $\frac{1}{2}(n+3 \bar{\delta}(G)+1-t)=n-\frac{1}{2}$. Theorem 3(a) gives $c(G) \geq\left\lceil\min \left\{n, n-\frac{1}{2}, n+\mathrm{NC}_{t}(G)-\alpha(G)\right\}\right\rceil=\min \left\{n, n+\mathrm{NC}_{t}(G)\right.$ $-\alpha(G)\}$.

(b) The proof of (b) is completely analogous to the proof of (a).

If $G$ is a graph on $n$ vertices and $t \in \mathbb{N}$ such that $t \leq \alpha(G)$, then clearly $\mathrm{NC}_{t}(G) \leq n-\alpha(G)$, hence $2 \mathrm{NC}_{t}(G) \leq n+\mathrm{NC}_{t}(G)-\alpha(G)$. So Theorem 3 has the following consequences.

\section{Corollary 5.}

(a) If $G \in G_{2}(n)$ and $t \in \mathbb{N}$, then $c(G) \geq \min \left\{n, \frac{1}{2}(n+3 \bar{\delta}(G)+1-t), 2 \mathrm{NC}_{t}(G)\right\}$.

(b) If $G \in G_{1}(n)$ and $t \in \mathbb{N}$, then $c(G) \geq \min \left\{n, \frac{1}{2}(n+3 \bar{\delta}(G)+4-t), 2 \mathrm{NC}_{t}(G)\right\}$.

Corollary 6 (Broersma, Van den Heuvel and Veldman [6]).

(a) If $G \in G_{2}(n)$, then $c(G) \geq \min \left\{n, 2 \mathrm{NC}_{3} \bar{\delta}(G)-n+2(G)\right\}$.

(b) If $G \in G_{1}(n)$, then $c(G) \geq \min \left\{n, 2 \mathrm{NC}_{3} \bar{\delta}(G)-n+5(G)\right\}$. 
Like Theorems 2 and 3, Corollaries 5 and 6 would not be true if the lower bounds on $\sigma_{3}(G)$ in the definitions of $G_{2}(n)$ or $\mathcal{G}_{1}(n)$ were relaxed. Also, Corollaries 5(a) and 6(a) are sharp in the sense that longer cycles are not implied by the hypothesis, as shown by suitable complete bipartite graphs. However, in contrast with Theorem 3, the subscript of $\mathrm{NC}(G)$ in the corollaries can be improved, as is shown in the next theorem, which will be proved in Section 5.

Theorem 7. If $G \in G_{2}(n) \cup G_{1}(n)$ and $t \in \mathbb{N}$, then

$$
c(G) \geq \min \left\{n, \frac{1}{3}(2 n+4 \bar{\delta}(G)+1-2 t), 2 \mathrm{NC}_{t}(G)\right\} .
$$

We first show that Theorem 7 is a full generalization of Corollary 5(a). Both results give $c(G) \geq 2 \mathrm{NC}_{3}(G) \geq 2\left\lceil\frac{1}{3} \sigma_{3}(G)\right\rceil=2 \bar{\delta}(G)$. Hence it suffices to show that

$$
\left\lceil\frac{1}{3}(2 n+4 \bar{\delta}(G)+1-2 t)\right\rceil \geq\left\lceil\frac{1}{2}(n+3 \bar{\delta}(G)+1-t)\right\rceil,
$$

whenever $t$ satisfies $\frac{1}{2}(n+3 \bar{\delta}(G)+1-t)>2 \bar{\delta}(G)$. The latter inequality is equivalent to $t<n-\bar{\delta}(G)+1$ and hence to $t \leq n-\bar{\delta}(G)$, which does indeed imply (2).

Theorem 7 does not completely generalize Corollary $5\left(\right.$ b). If $\sigma_{3}(G) \leq \frac{3}{2}(n-13)$, hence $\bar{\delta}(G) \leq\left[\frac{1}{2}(n-13)\right] \leq \frac{1}{2}(n-13)+\frac{1}{2}=\frac{1}{2} n-6$, then $n-\bar{\delta}(G)-7 \geq \frac{1}{2} n-1$. Since $\mathrm{NC}_{[n / 2\rceil-1}(G) \geq\left\lceil\frac{1}{2} n\right\rceil$ for a 1-tough graph $G$ on $n$ vertices, this means that if $\sigma_{3}(G) \leq \frac{3}{2}(n-$ 13), then the best lower bound for $c(G)$ in both Corollary 5(b) and Theorem 7 will occur for $t \leq n-\bar{\delta}(G)-8$, which implies $\left\lceil\frac{1}{3}(2 n+4 \bar{\delta}(G)+1-2 t)\right\rceil \geq\left\lceil\frac{1}{2}(n+3 \bar{\delta}(G)+4-t)\right\rceil$. So Theorem 7 generalizes Corollary $5($ b $)$ if $\sigma_{3}(G) \leq \frac{3}{2}(n-13)$.

Note that if $G \in G_{1}(n)$ satisfies the hypothesis of Theorem 7 with $\sigma_{3}(G) \geq \frac{3}{2} n-1$, then $G$ is hamiltonian. This follows from $2 \mathrm{NC}_{3}(G) \geq 2 \cdot \frac{1}{3} \sigma_{3}(G) \geq n-\frac{2}{3}$ and, since $n \geq 3$ and $\bar{\delta}(G) \geq\left\lceil\frac{1}{3}\left(\frac{3}{2} n-1\right)\right\rceil=\left\lceil\frac{1}{2} n-\frac{1}{3}\right\rceil=\left\lceil\frac{1}{2} n\right\rceil$,

$$
\frac{1}{3}(2 n+4 \bar{\delta}(G)+1-2 \cdot 3) \geq \frac{1}{3}(4 n-5)>n-1 .
$$

Next we show that both the lower bound on $c(G)$ and the subscript of $\mathrm{NC}(G)$ in the conclusion of Theorem 7 cannot be increased in general if $G \in \mathcal{G}_{2}(n)$. This is shown by the graphs $G_{k, \ell, m}$ defined after Theorem 3. For $t=\ell+\left\lfloor\frac{1}{2} m\right\rfloor$ we have

$$
\begin{aligned}
\frac{1}{3}\left(2 n+4 \bar{\delta}\left(G_{k, \ell, m}\right)+1-2 t\right) & \geq 2 k+m+\frac{1}{3}>c\left(G_{k, \ell, m}\right), \\
2 \mathrm{NC}_{t}\left(G_{k, \ell, m}\right) & \leq 2 k+m=c\left(G_{k, \ell, m}\right),
\end{aligned}
$$

and for $t=\ell+\left\lfloor\frac{1}{2} m\right\rfloor+1$ we obtain

$$
\begin{aligned}
\frac{1}{3}(2 n+4 \bar{\delta}(G)+1-2 t) & \leq 2 k+m=c\left(G_{k, \ell, m}\right), \\
2 \mathrm{NC}_{t}\left(G_{k, \ell, m}\right) & \geq 2 k+m+1>c\left(G_{k, \ell, m}\right) .
\end{aligned}
$$

Hence Theorem 7 is best possible if $G \in G_{2}(n)$ and $\sigma_{3}(G) \equiv 0(\bmod 3)$. Again, by deleting one or two suitable edges of $G_{k, \ell, m}$, we can prove it is best possible for $\sigma_{3}(G) \not \equiv 0(\bmod 3)$.

We do not believe that Theorem 7 is best possible for $G \in G_{1}(n)$. A possible improvement will be discussed in Section 2. 
Corollary 8. If $G \in G_{2}(n) \cup G_{1}(n)$ and $t \in \mathbb{N}$ with $t \leq \frac{1}{2}(4 \bar{\delta}(G)-n+3)$, then $c(G) \geq$ $\min \left\{n, 2 \mathrm{NC}_{l}(G)\right\}$.

Proof We have $\frac{1}{3}(2 n+4 \bar{\delta}(G)+1-2 t) \geq \frac{1}{3}(3 n-2)=n-\frac{2}{3}$. So by Theorem 7 , $c(G) \geq \min \left\{n, n-\frac{2}{3}, 2 \mathrm{NC}_{t}(G)\right\}$. We can conclude $c(G) \geq \min \left\{n, 2 \mathrm{NC}_{t}(G)\right\}$.

The result in Corollary 8 for $G \in G_{2}(n)$ was conjectured in [6]. There a weaker version with $t \leq \frac{1}{8}\left(6 \sigma_{3}(G)-5 n+17\right)$ was proved.

Theorem 7 also generalizes the following result.

Corollary 9 (Häggkvist [9]). Let $G$ be a nonhamiltonian 2-connected graph on $n$ vertices such that $\delta(G) \geq \frac{1}{3}(n+2)$ and let $t=2 \delta(G)-\left\lceil\frac{1}{2}(n+1)\right\rceil+2$. Then $\mathrm{NC}_{t}(G) \leq \frac{1}{2}(n-1)$.

Proof. We have $\sigma_{3}(G) \geq 3 \delta(G) \geq n+2$. Furthermore, $t \leq 2 \delta(G)-\frac{1}{2}(n+1)+2 \leq$ $\frac{1}{2}(4 \bar{\delta}(G)-n+3)$, since $\delta(G) \leq \bar{\delta}(G)$. By Corollary 8 this means $c(G) \geq \min \left\{n, 2 \mathrm{NC}_{t}(G)\right\}$. Since $G$ is nonhamiltonian, $c(G) \leq n-1$, hence $\mathrm{NC}_{t}(G) \leq \frac{1}{2}(n-1)$.

The remainder of this paper is organized as follows. In Section 2 we discuss some possible strengthenings of our results for 1-tough graphs. In Sections 3 and 4 we prove and cite some preliminary results. More particularly, in Section 3 we prove a variant of the Hopping Lemma from Woodall [14]. The main results, Theorems 3 and 7, are proved in Section 5.

\section{SOME CONJECTURES ON 1-TOUGH GRAPHS}

In this section we discuss some possible strengthenings of the results for 1-tough graphs.

Conjecture 10. If $G \in G_{1}(n)$ and $t \in \mathbb{N}$, then

$$
c(G) \geq \min \left\{n, \frac{1}{4}(3 n+3 \bar{\delta}(G)+2-t), n+\frac{1}{2}\left(\mathrm{NC}_{t}(G)-\alpha(G)-1\right)\right\} .
$$

Conjecture 10, if true, is a full generalization of Theorem 3(b). This follows from the fact that for $t \geq 3 \bar{\delta}(G)-n+6$ we have $\frac{1}{4}(3 n+3 \bar{\delta}(G)+2-t) \geq \frac{1}{2}(n+3 \bar{\delta}(G)+$ $4-t)$, for $t \leq 3 \bar{\delta}(G)-n+5$ we have $\frac{1}{2}(n+3 \bar{\delta}(G)+4-t) \geq n-\frac{1}{2}$ and $\frac{1}{4}(3 n+$ $3 \bar{\delta}(G)+2-t) \geq n-\frac{3}{4}$, for $\alpha(G) \geq \mathrm{NC}_{t}(G)+1$, we have $n+\frac{1}{2}\left(\mathrm{NC}_{t}(G)-\alpha(G)-\right.$ 1) $\geq n+\mathrm{NC}_{t}(G)-\alpha(G)$, and for $\alpha(G) \leq \mathrm{NC}_{t}(G)$ we have $n+\mathrm{NC}_{t}(G)-\alpha(G) \geq n$ and $n+\frac{1}{2}\left(\mathrm{NC}_{t}(G)-\alpha(G)-1\right) \geq n-\frac{1}{2}$. So we obtain that the lower bound on $c(G)$ in Conjecture 10 is always greater than or equal to the lower bound in Theorem 3(b).

If true, Conjecture 10 is best possible, as shown by the following examples. For $m \in \mathbb{N}$, let $F_{m}$ be the graph obtained from $K_{2 m+1}+\overline{K_{2 m+1}}$ by adding the edges of a matching between $K_{2 m+1}$ and $\overline{K_{2 m+1}}$. For $k, \ell, m \in \mathbb{N}$ with $k \geq \ell+1 \geq 4 m+1$, define the 1 tough graph $H_{k, \ell, m}$ as the join of $K_{k}$ and $\ell K_{1}+(k-\ell-1) K_{2}+F_{m}$ (see Fig. 2). We have $n=\left|V\left(H_{k, \ell, m}\right)\right|=3 k-\ell+4 m, \sigma_{3}\left(H_{k, \ell, m}\right)=3 k \geq n, \alpha\left(H_{k, \ell, m}\right)=k+2 m$ and $c\left(H_{k, \ell, m}\right)=3 k-\ell+3 m$. Furthermore, $\bar{\delta}\left(H_{k, \ell, m}\right)=k$ and $\mathrm{NC}_{\ell+x}\left(H_{k, \ell, m}\right)=k+x$ if $0 \leq$ $x \leq k-\ell+2 m$. This means that for $t=\ell+1$ we have

$$
\begin{gathered}
\frac{1}{4}\left(3 n+3 \bar{\delta}\left(H_{k, \ell, m}\right)+2-t\right)=3 k-\ell+3 m+\frac{1}{4}>c\left(H_{k, \ell, m}\right), \\
n+\frac{1}{2}\left(\mathrm{NC}_{t}\left(H_{k, \ell, m}\right)-\alpha\left(H_{k, \ell, m}\right)-1\right)=3 k-\ell+3 m=c\left(H_{k, \ell, m}\right),
\end{gathered}
$$




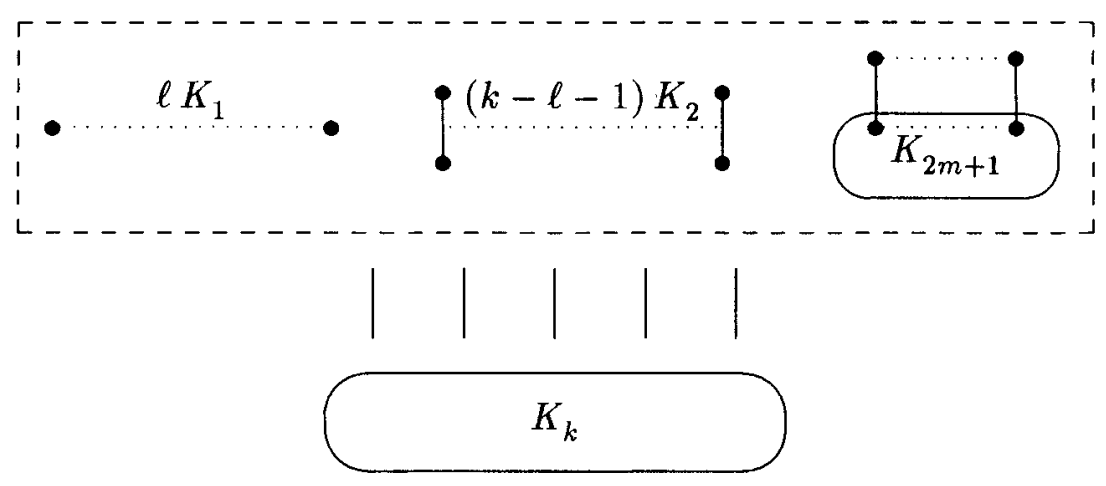

FIGURE 2. The graph $H_{k, \ell, m}$. The vertical lines indicate a join.

and for $t=\ell+2$ we obtain

$$
\begin{aligned}
& \frac{1}{4}\left(3 n+3 \bar{\delta}\left(H_{k, \ell, m}\right)+2-t\right)=3 k-\ell+3 m=c\left(H_{k, \ell, m}\right), \\
& n+\frac{1}{2}\left(\mathrm{NC}_{t}\left(H_{k, \ell, m}\right)-\alpha\left(H_{k, \ell, m}\right)-1\right)=3 k-\ell+3 m+\frac{1}{2}
\end{aligned}
$$$$
>c\left(H_{k, \ell, m}\right)
$$

If Conjecture 10 is true, then this implies the truth of the following conjectures, in the same way as Corollaries 4(b) and 5(b) are consequences of Theorem 3(b).

Conjecture 11. If $G \in G_{1}(n)$, then $c(G) \geq \min \left\{n, n+\frac{1}{2}\left(\mathrm{NC}_{3} \bar{\delta}(G)-n+5(G)-\alpha(G)-1\right)\right\}$.

Conjecture 12. If $G \in G_{1}(n)$ and $t \in \mathbb{N}$, then $c(G) \geq \min \left\{n, \frac{1}{4}(3 n+3 \bar{\delta}(G)+2-\right.$ t), $\left.\frac{1}{2}(n-1)+\mathrm{NC}_{t}(G)\right\}$.

The consequence of Conjecture 10 that is the analog of Corollary $6(\mathrm{~b})$, reads that a graph $G \in$ $G_{1}(n)$ satisfies $c(G) \geq \min \left\{n, \frac{1}{2}(n-1)+\mathrm{NC}_{3 \bar{\delta}(G)-n+5}(G)\right\}$. But this immediately follows from Corollary 4(b), since for a 1 -tough graph $G$ on $n \geq 3$ vertices we have $\alpha(G) \leq \frac{1}{2} n$.

We do not believe that Conjecture 12 is best possible. In fact, we conjecture the following.

Conjecture 13. If $G \in G_{1}(n)$ and $t \in \mathbb{N}$, then $c(G) \geq \min \left\{n, \frac{1}{6}(5 n+4 \bar{\delta}(G)+2-\right.$ $\left.2 t), \frac{1}{2}(n-1)+\mathrm{NC}_{t}(G)\right\}$.

If true, Conjecture 13 is best possible as can be seen by considering the graphs $H_{k, \ell, m}$ defined above, and the possibilities $t=\ell+m+\left\lfloor\frac{1}{2}(k-\ell+1)\right\rfloor$ and $t=\ell+m+\left\lfloor\frac{1}{2}(k-\ell+\right.$ $1)\rfloor+1$. The details are left to the reader. Conjecture 13 also means an improvement of Theorem 7.

In the same way as Corollary 8 follows from Theorem 7 , the truth of the following conjecture follows from the truth of Conjecture 13 .

Conjecture 14. If $G \in G_{1}(n)$ and $t \in \mathbb{N}$ with $t \leq \frac{1}{2}(4 \bar{\delta}(G)-n+7)$, then $c(G) \geq$ $\min \left\{n, \frac{1}{2}(n-1)+\mathrm{NC}_{t}(G)\right\}$.

Let $G \in G_{1}(n)$ and set $t=\bar{\delta}(G)+1$. Then we have $\frac{1}{4}(3 n+3 \bar{\delta}(G)+2-t)=\frac{1}{4}(3 n+$ $1)+\frac{1}{2} \bar{\delta}(G)$. Also, $\mathrm{NC}_{t}(G) \geq t+1$ and $\alpha(G) \leq \frac{1}{2} n$, hence $n+\frac{1}{2}\left(\mathrm{NC}_{t}(G)-\alpha(G)-\right.$ 
1) $\geq \frac{1}{4}(3 n+2)+\frac{1}{2} \bar{\delta}(G)$. This shows that the truth of Conjecture 10 implies the truth of the following conjecture.

Conjecture 15. (Bauer, Morgana, Schmeichel, and Veldman [2]). If $G \in G_{1}(n)$, then $c(G) \geq$ $\min \left\{n, \frac{1}{4}(3 n+1)+\frac{1}{2} \bar{\delta}(G)\right\} \geq \frac{1}{12}(11 n+3)$.

Setting $t=\bar{\delta}(G)+1$ and $\alpha(G) \leq \frac{1}{2} n$ in Theorem 3(b) we obtain the following result.

Conjecture 16. If $G \in \mathcal{G}_{1}(n)$, then $c(G) \geq \min \left\{n, \frac{1}{2}(n+3)+\bar{\delta}(G)\right\} \geq \min \left\{n, \frac{1}{6}(5 n+9)\right\}$.

Recently, the following partial improvement of Corollary 16 was proved, as a first step toward a proof of Conjecture 15.

Theorem $17(\mathbf{L i}[11])$. If $G$ is a 1-tough graph on $n$ vertices such that $\frac{1}{3} n \leq \delta(G) \leq$ $\frac{1}{2}(n-7)$, then

$$
c(G) \geq \min \left\{n, \frac{1}{3}(2 n+1+2 \delta(G))\right\} \geq \frac{1}{9}(8 n+3) .
$$

\section{A HOPPING LEMMA USING ALL VERTICES OUTSIDE THE CYCLE}

The Hopping Lemma was obtained in Woodall [14] as a tool for problems concerning paths and cycles in graphs. In this section we prove a version of it for graphs $G$ that satisfy $c(G) \geq p(G)$. This is stronger than the condition imposed on $G$ in the original Hopping Lemma, and this results in a stronger conclusion.

We need some additional terminology and notation. A cycle $C$ in a graph $G$ is called a dominating cycle if $G-V(C)$ contains no edges. By $\vec{C}$ we denote the cycle $C$ with a given orientation, and by $\bar{C}$ the cycle with the reverse orientation. If $u, v \in V(C)$, then $u \vec{C} v$ denotes the consecutive vertices of $C$ from $u$ to $v$ in the direction specified by $\vec{C}$. The same vertices, in reverse order, are given by $v \bar{C} u$. We will consider $u \vec{C} v$ and $v \grave{C} u$ both as paths and as vertex sets. If $u \in V(C)$, then $u^{+}$denotes the successor of $u$ on $\vec{C}$, and $u^{-}$denotes its predecessor. If $U \subseteq V(C)$, then $U^{+}=\left\{u^{+} \mid u \in U\right\}$ and $U^{-}=\left\{u^{-} \mid u \in U\right\}$. When more than one cycle is under consideration, we sometimes write $u^{+C}, u^{-C}, \ldots$ instead of just $u^{+}, u^{-}, \ldots$ in order to avoid ambiguity.

Similar notation as described above is used for paths. We will use the notation $P=v_{1} \vec{P} v_{p}$ to say that $P$ is a path with origin $v_{1}$, terminus $v_{p}$, and orientation from $v_{1}$ to $v_{p}$.

In the remainder of this section let $G$ be a nonhamiltonian connected graph with $c(G) \geq p(G)$. Clearly this implies that every longest cycle in $G$ is a dominating cycle. Let $C$ be a longest cycle in $G$ and set $R=V(G)-V(C)$. Fix an orientation $\vec{C}$ on $C$. Set $Y_{0}=\varnothing$ and for $i \geq 1$,

$$
X_{i}=N\left(Y_{i-1} \cup R\right) \cap V(C), \quad Y_{i}=X_{i}^{+} \cap X_{i}^{-} .
$$

Set $X=\bigcup_{i=1}^{\infty} X_{i}$ and $Y=\bigcup_{i=1}^{\infty} Y_{i}$.

Lemma 18. The sets $X$ and $Y$ satisfy

(a) $X \cap X^{+}=\varnothing$ and $X \cap Y=\varnothing$;

(b) $N(Y) \subseteq X$;

(c) $Y$ is an independent set. 
For the proof of Lemma 18 we use the notion of a hopping path. If $x \in X$, then the height $h(x)$ of $x$ is defined by $h(x)=\min \left\{i \mid x \in X_{i}\right\}$. A path $P=x_{1} \vec{P} x_{2}$ is called a hopping path if it satisfies the following requirements:

(HP1) $x_{1}, x_{2} \in X$

(HP2) $V(P)=V(C)$;

(HP3) if $i<\max \left\{h\left(x_{1}\right), h\left(x_{2}\right)\right\}$ and $y \in Y_{i}-\left\{x_{1}, x_{2}\right\}$, then $y^{-P}, y^{+P} \in X_{i}$.

The height $h(P)$ of a hopping path $P=x_{1} \vec{P} x_{2}$ is defined by $h(P)=\max \left\{h\left(x_{1}\right), h\left(x_{2}\right)\right\}$.

Lemma 19. If there exists a hopping path, then there exists a hopping path of height 1.

Proof Let $P=x_{1} \vec{P} x_{2}$ be a hopping path, chosen such that

(i) $h(P)$ is minimum, and

(ii) $h\left(x_{1}\right)+h\left(x_{2}\right)$ is minimum, subject to (i).

Without loss of generality, we may assume $h\left(x_{1}\right) \geq h\left(x_{2}\right)$. If $h(P)=1$, we are done, so assume $h(P)=i+1 \geq 2$. We distinguish two cases, in each of which we reach a contradiction.

Case 1. $h\left(x_{2}\right)<h\left(x_{1}\right)=i+1$.

Then $x_{1} \in X_{i+1}-X_{i}$ and hence there exists a vertex $y \in Y_{i}-Y_{i-1}$ with $x_{1} y \in E(G)$. If $y=x_{2}$, then $y \in X_{i} \cap Y_{i}$, so, by the definition of $Y_{i}$, also $y^{+C} \in X_{i}$. Then the path $y^{+C} \vec{C} y$ is a hopping path containing all vertices of $V(C)$ and satisfies $h\left(y^{+C} \vec{C} y\right) \leq i \leq h(P)$, contradicting the choice of $P$. So we have $y \neq x_{1}, x_{2}$. By (HP2) and (HP3) this means $y \in V(P)$ and $y^{-P}, y^{+P} \in X_{i}$. Set $Q=y^{-P} x_{1} y \vec{P} x_{2}$, then $Q$ satisfies (HP1). Since $V(Q)=$ $V(P), Q$ also satisfies (HP2). We have $x_{1} \notin Y_{i-1}$, otherwise $y \in X_{i} \cap Y_{i}$ and we reach a contradiction as in the case $x_{2} \in Y_{i}$, and $y \notin Y_{i-1}$. Finally, $\left\{v^{-P}, v^{+P}\right\}=\left\{v^{-Q}, v^{+Q}\right\}$ for each internal vertex $v$ of $Q$ with $v \neq x_{1}, y$. It follows that $Q$ also satisfies (HP3), whence $Q$ is a hopping path with $h(Q) \leq i<h(P)$, contradicting the chose of $P$.

Case 2. $h\left(x_{1}\right)=h\left(x_{2}\right)=i+1$.

In this case there exist vertices $y_{1}, y_{2} \in Y_{i}-Y_{i-1}$ with $x_{1} y_{1}, x_{2} y_{2} \in E(G)$. If $y_{1}=x_{2}$, then $y_{1} \in Y_{i} \cap X_{i+1}$, so, by the definition of $Y_{i}, y_{1}^{+C} \in X_{i}$. Then the path $y_{1}^{+C} \vec{C} y_{1}$ is a hopping path that satisfies $h\left(y_{1}^{+C} \vec{C} y_{1}\right) \leq h(P)$ and $h\left(y_{1}^{+C}\right)+h\left(y_{1}\right)<h\left(x_{1}\right)+h\left(x_{2}\right)$, contradicting the choice of $P$. So we have $y_{1} \neq x_{2}$ and, similarly, $y_{2} \neq x_{1}$. Now if $y_{1} \in x_{1} \vec{P} y_{2}$ set

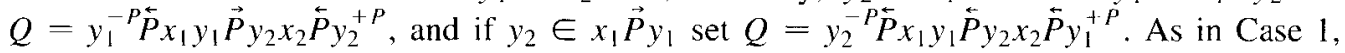
$Q$ is a hopping path with $h(Q)<h(P)$, contradicting the choice of $P$.

Lemma 20. There exists no hopping path.

Proof. Suppose $P=x_{1} \vec{P} x_{2}$ is a hopping path. By Lemma 19 we may assume $h(P)=1$. Since $x_{1}, x_{2} \in X_{1}$, we have $r_{1} x_{1} \in E(G)$ and $r_{2} x_{2} \in E(G)$ for some $r_{1}, r_{2} \in R$. If $r_{1}=r_{2}$, then $r_{1} x_{1} \vec{P} x_{2} r_{1}$ is a cycle of length $|V(C)|+1$, contradicting the choice of $C$; and if $r_{1} \neq r_{2}$, then $r_{1} x_{1} \vec{P} x_{2} r_{2}$ is a path of length $|V(C)|+1$, contradicting $|V(C)|=c(G) \geq p(G)$.

Now we can prove the statements in Lemma 18.

Proof of Lemma 18. (a) If $x \in X \cap X^{+}$or $x \in X \cap Y$, then $x^{-} \in X$, hence $x \vec{C} x^{-}$is a hopping path, contradicting Lemma 20.

(b) By the definition of $X$ we have $N(R) \subseteq X$ and $N(Y) \cap V(C) \subseteq X$. Since $N(R) \cap$ $Y=\varnothing$ by (a), it follows that $N(Y) \cap R=\varnothing$, hence $N(Y) \subseteq V(C)$. This means $N(Y) \subseteq X$. 
(c) This is an immediate consequence of (a) and (b).

Set $U=X^{+}-Y$ and $W=X^{-}-Y$. Let $u_{1}, \ldots, u_{1}$ be the vertices of $U$, occurring on $\vec{C}$ in the order of their indices. For $i=1, \ldots, t$, let $w_{i}$ be the unique vertex of $W$ in the component of $C-X$ containing $u_{i}$.

\section{Corollary 21.}

(a) $U$ and $W$ are independent sets.

(b) If $v \in w_{j}^{++} \vec{C} u_{i}^{--}$and $u_{i} v \in E(G)$, then $w_{j} v^{-} \notin E(G)$ and $w_{j} v^{+} \notin E(G)(i, j=$ $1, \ldots, t, i \neq j+1(\bmod t))$.

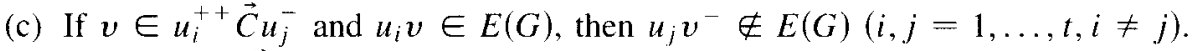

(d) If $v \in w_{i}^{++} \vec{C} w_{j}^{-}$and $w_{i} v \in E(G)$, then $w_{j} v^{-} \notin E(G)(i, j=1, \ldots, t, i \neq j)$.

Proof. (a) We only prove that $U$ is an independent set, the proof for $W$ is similar. Suppose $u_{i} u_{j} \in E(G)$ for some $i \neq j$. Then, since $u_{i}, u_{j}, \notin Y$, the path $u_{i}^{-} \overleftarrow{C} u_{j} u_{i} \vec{C} u_{j}^{-}$is a hopping path, contradicting Lemma 20 .

(b) If $v \in w_{j}^{++} \vec{C} u_{i}^{--}$and $u_{i} v, w_{j} v^{-} \in E(G)$, then we have $v, v^{-} \notin Y$. So the path $u_{i}^{-} \overleftarrow{C} v u_{i} \vec{C} w_{j} v^{-} \bar{C} w_{j}^{+}$is a hopping path, a contradiction. The rest of (b) is proved similarly.

(c) Suppose $v \in u_{i}^{++} \vec{C} u_{j}^{-}$and $u_{i} v, u_{j} v^{-} \in E(G)(i \neq j)$. Since $u_{i}, u_{j}, v, v^{-} \notin Y$, we obtain the hopping path $u_{i}^{-} \stackrel{\leftarrow}{C} u_{j} v^{-} \overleftarrow{C} u_{i} v \vec{C} u_{j}^{-}$, a contradiction.

(d) The proof of (d) is similar to the proof of (c).

The results in this section were proved in Min Aung [12] for graphs satisfying a different condition. In the remainder of the paper we only use Corollary $21(\mathrm{a})$. We choose to give Corollary 21(b)-(d) as well, in order to make it possible to compare our results with known extensions of the original Hopping Lemma (see, e.g., Jackson [10]).

\section{FURTHER PRELIMINARY RESULTS}

The following results, together with Lemma 18 and Corollary 21(a), play an essential role throughout the proofs of the main results of this paper. If a cycle $C$ in a graph $G$ is not a Hamilton cycle, then we define $\mu(C)=\max \{d(v) \mid v \in V(G)-V(C)\}$.

Theorem 22. Let $G \in G_{2}(n) \cup G_{1}(n)$. Then we have

(i) $c(G) \geq p(G)$;

(ii) every longest cycle in $G$ is a dominating cycle;

(iii) if $G$ is nonhamiltonian, then $G$ contains a longest cycle $C$ with $\mu(C) \geq \frac{1}{3} \sigma_{3}(G)$.

Proof. Part (i) is in Enomoto, Van den Heuvel, Kaneko and Saito [8]. Part (ii) follows immediately from (i); also, (ii) is in Bondy [4] for $G \in \mathcal{G}_{2}(n)$ and in Bauer, Morgana, Schmeichel, and Veldman [2] for $G \in G_{1}(n)$. Part (iii) is implicit in the proofs of [2, Theorems 9 and 10]. Parts (ii) and (iii) also appear in Bauer, Broersma, and Veldman [1].

If $C$ is a cycle in a graph, with orientation $\vec{C}$, and $B \subseteq V(C)$ with $B \cap B^{+}=\varnothing$, then the components of $C-B$ will be called the B-segments of $C$. A $B$-segment is a $B-p$-segment if it contains $p$ vertices. 
Lemma 23. Let $C$ be a cycle in a graph, with orientation $\vec{C}$, and $B \subseteq V(C)$ with $B \cap B^{+}=$ $\varnothing$. Set $Z_{B}=V(C)-\left(B \cup B^{+} \cup B^{-}\right)$and let $b_{1}$ denote the number of $B-1$-segments on $C$. Then $b_{1}=3|B|+\left|Z_{B}\right|-|V(C)| \geq 3|B|-|V(C)|$.

Proof. There are exactly $|B| B$-segments. The number of $B$-segments with two or more vertices is equal to $|B|-b_{1}$. So we have $|V(C)|=|B|+b_{1}+2\left(|B|-b_{1}\right)+\left|Z_{B}\right|=$ $3|B|-b_{1}+\left|Z_{B}\right|$, hence $b_{1}=3|B|+\left|Z_{B}\right|-|V(C)|$.

For the remainder of this section, let $G$ be a nonhamiltonian graph in $G_{2}(n)$ or $G_{1}(n)$. Let $C$ be a longest cycle of $G$ for which $\mu(C)$ is maximal, and fix an orientation $\vec{C}$ on $C$. By Theorem 22 (i), $c(G) \geq p(G)$, and so we can use the variant of the Hopping Lemma established in Section 3. Define $R, X$, and $Y$ as in Section 3. Let $y_{0} \in V(G)-V(C)$ with $d\left(y_{0}\right)=\mu(C)$, and let

$$
A=N\left(y_{0}\right) \subseteq N(R) \subseteq X \subseteq V(C)
$$

by Theorem 22 (ii) and the definition of $X$. By (3) and Theorem 22 (iii) we have

$$
|X| \geq|A|=d\left(y_{0}\right) \geq\left\lceil\frac{1}{3} \sigma_{3}(G)\right\rceil=\bar{\delta}(G)
$$

Let $a_{1}$ denote the number of $A-1$-segments on $C$.

Lemma 24. We have $a_{1} \geq 3 \bar{\delta}(G)-n+|R|$, with equality if and only if $d\left(y_{0}\right)=\bar{\delta}(G)$ and, apart from the $A-1$-segments, $C$ contains $A-2$-segments only.

Proof. Since $C$ is a longest cycle, $A \cap A^{+}=\varnothing$. By Lemma 23 and (4), $a_{1} \geq 3|A|-$ $|V(C)| \geq 3 \bar{\delta}(G)-|V(C)|$, with equality if and only if $|A|=\bar{\delta}(G)$ and $\mid V(C)-\left(A \cup A^{+} \cup\right.$ $\left.A^{-}\right) \mid=0$. The result follows.

Lemma 25. There exists an $A-1$-segment $y_{1}$ such that $d\left(y_{1}\right) \geq \bar{\delta}(G)$.

Proof. Label the $A-1$-segments as $y_{1}, y_{2}, \ldots$ with $d\left(y_{1}\right) \geq d\left(y_{2}\right) \geq \cdots$. If $a_{1} \geq 3$, then $\left\{y_{1}, y_{2}, y_{3}\right\}$ is an independent set. So we have $d\left(y_{1}\right)+d\left(y_{2}\right)+d\left(y_{3}\right) \geq \sigma_{3}(G)$, which means $d\left(y_{1}\right) \geq \bar{\delta}(G)$. If $a_{1}=2$, then by (4) and Lemma $23, \bar{\delta}(G) \leq|A| \leq \frac{1}{3}\left(a_{1}+n-|R|\right) \leq$ $\frac{1}{3}(n+1)$. Suppose $d\left(y_{1}\right)<\bar{\delta}(G)$. Since $d\left(y_{1}\right)$ is an integer, $d\left(y_{2}\right) \leq d\left(y_{1}\right) \leq \bar{\delta}(G)-1$. Thus we have

$$
\begin{aligned}
n & \leq \sigma_{3}(G) \leq d\left(y_{0}\right)+d\left(y_{1}\right)+d\left(y_{2}\right) \leq \frac{1}{3}(n+1)+2(\bar{\delta}(G)-1) \\
& \leq n-1,
\end{aligned}
$$

a contradiction.

So we can assume $a_{1} \leq 1$. By Lemma 24, (1) and the definitions of $G_{2}(n)$ and $G_{1}(n)$, $a_{1} \geq \sigma_{3}(G)-n+|R| \geq 1$, and equality implies $G \in G_{1}(n), 3 \bar{\delta}(G)=\sigma_{3}(G)=n,|R|=1$ and $C$ contains $A-1$-segments and $A-2$-segments only. Lemma 23 and (4) now give $d\left(y_{0}\right)=|A|=\frac{1}{3}(1+n-1)=\frac{1}{3} \sigma_{3}(G)$. Since $y_{1}^{+} \vec{C} y_{1}^{-} y_{0} y_{1}^{+}$is a longest cycle not containing $y_{1}$, we have $d\left(y_{1}\right) \leq d\left(y_{0}\right)$ by the choice of $C$. Let $x_{1}, \ldots, x_{|A|}$ be the vertices of $A$ in order round $\vec{C}$. Since $C$ is a longest cycle, clearly $\left\{y_{0}\right\} \cup A^{+}$and $\left\{y_{0}\right\} \cup A^{-}$are independent sets. Since $G$ is 1-tough, $\omega(G-A) \leq|A|$. Hence there exist two $A-2$-segments $x_{i}^{+} x_{i+1}^{-}$and $x_{j}^{+} x_{j+1}^{-}$such that $x_{i}^{+} x_{j+1}^{-} \in E(G)$ (indices mod $|A|$ ), otherwise $\omega(G-A)=|A|+|R|>|A|$. 
Since $x_{i}^{+} x_{j+1}^{-} \overleftarrow{C} x_{i+1} y_{0} x_{j+1} \vec{C} x_{i}^{+}$is a longest cycle not containing $x_{i+1}^{-}$, we have $d\left(x_{i+1}^{-}\right) \leq d\left(y_{0}\right)$, by the choice of $C$. Obviously, $\left\{y_{0}, y_{1}, x_{i+1}^{-}\right\}$is an independent set. So we have

$$
\sigma_{3}(G) \leq d\left(y_{0}\right)+d\left(y_{1}\right)+d\left(x_{i+1}^{-}\right) \leq 3 d\left(y_{0}\right)=\sigma_{3}(G) .
$$

Thus $d\left(y_{1}\right)=\frac{1}{3} \sigma_{3}(G)=\bar{\delta}(G)$.

\section{PROOFS OF THEOREMS 3 AND 7}

Assume that $G$ is a nonhamiltonian graph in $\mathcal{G}_{2}(n)$ or $\mathcal{G}_{1}(n)$, and $t \in \mathbb{N}$. Let $C$ be a longest cycle of $G$ with orientation $\vec{C}$ such that $\mu(C)$ is maximal, and let $y_{0} \in V(G)-V(C)$ with $d\left(y_{0}\right)=\mu(C)$. Copy the notation and terminology used in former sections. In particular, define $R, X_{1}, X, Y, U, W, A$, and $a_{1}$ as in Sections 3 and 4 .

By Lemma 25 there exists an $A-1$-segment $y_{1} \in Y$ such that $d\left(y_{1}\right) \geq \bar{\delta}(G)$. Since the $A-1$-segments are elements of $Y$, we have, by Lemma 24 ,

$$
|Y| \geq 3 \bar{\delta}(G)-n+|R|
$$

By Lemma 18 (a), (b), (c) and the fact that $N(R) \subseteq X$ by (3), it follows that

$$
R \cup Y \text { is an independent set with } N(R \cup Y) \subseteq X \subseteq V(C) \text {. }
$$

Since $N(R) \subseteq X$ and $X^{+}=Y \cup U$, from Corollary 21 (a) and Lemmas 18 (a), (b), (c) we have

$$
R \cup X^{+} \text {is an independent set. }
$$

Let $Z=V(C)-(X \cup Y \cup U \cup W)=V(C)-\left(X \cup X^{+} \cup X^{-}\right)$. By Lemma 23 we have

$$
0 \leq|Z|=|V(C)|+|Y|-3|X|=n+|Y|-3|X|-|R| .
$$

Proof of Theorem 3(a). If $c(G) \geq \frac{1}{2}(n+3 \bar{\delta}(G)+1-t)$, then we are done. So we may assume $n-|R|=c(G) \leq \frac{1}{2}(n+3 \bar{\delta}(G)-t)$, which implies

$$
t \leq n+3 \bar{\delta}(G)-2(n-|R|)=3 \bar{\delta}(G)-n+2|R| .
$$

Set $S=Y \cup R$. By (5) this means

$$
|S|=|Y|+|R| \geq 3 \bar{\delta}(G)-n+2|R| \geq t
$$

By (6) and (7), both $S$ and $R \cup N(S)^{+}$are independent sets. So we obtain

$$
\begin{aligned}
\alpha(G) & \geq\left|R \cup N(S)^{+}\right|=|R|+\left|N(S)^{+}\right|=|V(G)|-|V(C)|+|N(S)| \\
& \geq n-|V(C)|+\mathrm{NC}_{|S|}(G) .
\end{aligned}
$$

Together with (9) this gives $c(G)=|V(C)| \geq n+\mathrm{NC}_{t}(G)-\alpha(G)$. 
Proof of Theorem 3(b). Again, we may assume that $c(G) \leq \frac{1}{2}(n+3 \bar{\delta}(G)+3-t)$, hence

$$
t \leq n+3 \bar{\delta}(G)+3-2 c(G)=3 \bar{\delta}(G)-n+2|R|+3
$$

Let $Y=\left\{y_{1}, \ldots, y_{|Y|}\right\}$, with $d\left(y_{2}\right) \geq d\left(y_{3}\right) \geq \ldots$. Let $u_{1}, \ldots, u_{|U|}$ be the vertices of $U$ in order round $\vec{C}$ such that $u_{1}$ is the first vertex of $U$ following $y_{1}$ on $\vec{C}$. For $i=1, \ldots,|U|$, let $w_{i}$ be the unique vertex of $W$ in the $X$-segment containing $u_{i}$.

We state some observations which will be used repeatedly. We will say that a property $\mathcal{P}$ of $G$ holds by (lca) (longest cycle argument) if the contrary to $\mathcal{P}$ implies the existence of a cycle $C^{\prime}$ longer than $C$. (lca) often represents an argument which is standard in hamiltonian graph theory. The cycle $C^{\prime}$ will often be given between brackets after the statement of $\mathcal{P}$.

(OB1) Let $y \in Y$ and assume $N\left(y_{0}\right)=N(y)=X$ and $u_{i} w_{j} \in E(G)$ for some $i \neq j$. Then $y \in w_{j} \vec{C} u_{i}, w_{i} y^{+} \notin E(G)$ and $w_{i} y^{-} \notin E(G)$.

Proof. By (lca) we have $y \in w_{j} \vec{C} u_{i}\left(y^{-} y_{0} w_{j}^{+} \vec{C} u_{i}^{-} y \vec{C} w_{j} u_{i} \vec{C} y^{-}\right)$. Also, $w_{i} y^{+} \notin E(G)$ $\left(y u_{i} \stackrel{\leftarrow}{C} y^{+} w_{i} \stackrel{\leftarrow}{C} u_{i} w \stackrel{\leftarrow}{C} w_{i}^{+} y_{0} w_{j}^{+} \vec{C} y\right)$ and $w_{i} y^{-} \notin E(G)\left(y \overleftrightarrow{C} u_{i}^{-} y_{0} w_{j}^{+} \vec{C} y^{-} w_{i} \stackrel{C}{C} u_{i} w_{j} \overleftarrow{C} w_{i}^{+} y\right)$.

(OB2) Assume $N\left(y_{0}\right)=N\left(y_{1}\right)=X$ and $u_{i} w_{i} \in E(G)$, where $i \neq j$ and $i$ is chosen minimal. Then $N\left(w_{i}\right) \cap(U \cup W) \subseteq\left\{u_{i}\right\}$.

Proof. By Corollary 21 (a) we have $N\left(w_{i}\right) \cap W=\varnothing$. The minimality of $i$ implies $u_{l} w_{i} \notin E(G)$ for $l=1, \ldots, i-1$. By (OB 1$)$, if $u_{l} \in N\left(w_{i}\right) \cap U$, then $y_{1} \in w_{i} \vec{C} u_{l}$, so also $u_{l} w_{i} \notin E(G)$ for $l=i+1, \ldots,|U|$.

(OB3) If $|X|=\bar{\delta}(G)$, then $d\left(y_{0}\right)=\cdots=d\left(y_{|Y|-\varepsilon}\right)=\bar{\delta}(G)$, where $\varepsilon=3 \bar{\delta}(G)-\sigma_{3}(G)$.

Proof. First note that by (5) we have $|Y|-\varepsilon \geq|R| \geq 1$. By (4), $d\left(y_{0}\right)=\bar{\delta}(G)$. For $y \in Y$ it yields $N(y) \subseteq X$, so $d(y) \leq|X|=\bar{\delta}(G)$. By the choice of $y_{1}$ we have $d\left(y_{1}\right) \geq$ $\bar{\delta}(G)$, hence $d\left(y_{1}\right)=\bar{\delta}(G)$ and we are done if $|Y|=1$. Now assume $|Y| \geq 2$ and $d\left(y_{|Y|-\varepsilon}\right) \leq$ $\bar{\delta}(G)-1$. By definition, $d\left(y_{2}\right) \geq d\left(y_{3}\right) \geq \cdots \geq d\left(y_{|Y|}\right)$, which gives the contradiction

$$
\begin{aligned}
\sigma_{3}(G) & \leq d\left(y_{|Y|-2}\right)+d\left(y_{|Y|-1}\right)+d\left(y_{|Y|}\right) \leq 3 \bar{\delta}(G)-\varepsilon-1 \\
& =\sigma_{3}(G)-1
\end{aligned}
$$

This shows $d\left(y_{|Y|-\varepsilon}\right) \geq \bar{\delta}(G)$, hence $d\left(y_{0}\right)=d\left(y_{1}\right)=\ldots=d\left(y_{|Y|-\varepsilon}\right)=\bar{\delta}(G)$.

A subset $S \subseteq V(G)$ will be called suitable if $|S| \geq t, N(S) \subseteq V(C)$ and both $S$ and $R \cup N(S)^{+}$are independent sets. If a suitable set $S$ exists, then we are done, since

$$
\begin{aligned}
\alpha(G) & \geq\left|R \cup N(S)^{+}\right|=|R|+\left|N(S)^{+}\right|=|V(G)|-|V(C)|+|N(S)| \\
& \geq n-|V(C)|+\mathrm{NC}_{|S|}(G),
\end{aligned}
$$

hence $c(G)=|V(C)| \geq n+\mathrm{NC}_{t}(G)-\alpha(G)$.

We will distinguish a number of cases, in each of which we either exhibit a suitable set, or reach a contradiction.

Case 1. $|Y|=3 \bar{\delta}(G)-n+|R|$.

By (4) and (8) we conclude $|Z|=0$ and $|X|=\bar{\delta}(G)$. So apart from the $X-1$-segments, $C$ contains $X-2$-segments only. Furthermore, we have $N\left(y_{0}\right)=N\left(y_{1}\right)=X$. Since $G$ is 
1-tough, $\omega(G-X) \leq|X|$. Hence there exist $i, j, i \neq j$, such that $u_{i} w_{j} \in E(G)$. Assume $i$ is chosen minimal. By (OB1) and (OB2), $N\left(w_{i}\right) \subseteq\left(X-\left\{y_{1}^{+}, y_{1}^{-}\right\}\right) \cup\left\{u_{i}\right\}$, so $d\left(w_{i}\right) \leq$ $\bar{\delta}(G)-1$. Now consider the greatest $h$ such that $u_{g} w_{h} \in E(G)$ for some $g \neq h$. By (OB1), $y_{1} \in w_{h} \vec{C} u_{g}$, so $i \leq g<h$. By (OB1) and (OB2) (now applied to $\overleftarrow{C}$ ), $N\left(u_{h}\right) \subseteq$ $\left(X-\left\{y_{1}^{+}, y_{1}^{-}\right\}\right) \cup\left\{w_{h}\right\}$, so $d\left(u_{h}\right) \leq \bar{\delta}(G)-1$. For $y \in Y$ we have $N(y) \subseteq X$, hence $d(y) \leq$ $\bar{\delta}(G)$. This gives

$$
n \leq \sigma_{3}(G) \leq d\left(w_{i}\right)+d\left(u_{h}\right)+d(y) \leq 3 \bar{\delta}(G)-2 .
$$

So we obtain $|Y| \geq|R|+2 \geq 3$ and $d(y)=\bar{\delta}(G)$ for all $y \in Y$. But then, in fact, we have $N\left(w_{i}\right) \subseteq\left(X-\bigcup_{y \in Y}\left\{y^{+}, y^{-}\right\}\right) \cup\left\{u_{i}\right\}$. Since $\left|\bigcup_{y \in Y}\left\{y^{+}, y^{--}\right\}\right| \geq|Y|+1$, this means $d\left(w_{i}\right) \leq \bar{\delta}(G)-|Y|$. Similarly, $d\left(u_{h}\right) \leq \bar{\delta}(G)-|Y|$. We reach the contradiction

$$
\sigma_{3}(G) \leq d\left(w_{i}\right)+d\left(u_{h}\right)+d\left(y_{1}\right) \leq 3 \bar{\delta}(G)-2|Y| \leq 3 \bar{\delta}(G)-6 .
$$

Case 2. $|Y|=3 \bar{\delta}(G)-n+|R|+1$.

By (4) and (8) we obtain $|X|=\bar{\delta}(G)$ and $|Z|=1$. So apart from the $X-1$ - and $X-$ 2-segments, $C$ contains exactly one $X-3$-segment, say $u_{l} u_{l}^{+} w_{l}$. Furthermore, we have $|Y|-\left(3 \bar{\delta}(G)-\sigma_{3}(G)\right) \geq|R|+1 \geq 2$, so by (OB3), $N\left(y_{0}\right)=N\left(y_{1}\right)=N\left(y_{2}\right)=X$. If $u_{l} w_{l} \in E(G)$, then, by (lca), $u_{l}^{+} u_{i} \notin E(G)$ for $i \neq l\left(u_{i} \vec{C} u_{l}^{-} y_{0} u_{i}^{-} \bar{C} w_{l} u_{l} u_{l}^{+} u_{i}\right)$ and $u_{l}^{+} w_{i} \notin$ $E(G)$ for $i \neq l\left(w_{i} \stackrel{\leftarrow}{C} w_{l}^{+} y_{0} w_{i}^{+} \vec{C} u_{l} w_{l} u_{l}^{+} w_{i}\right)$, hence $N\left(u_{l}^{+}\right) \subseteq X \cup\left\{u_{l}, w_{l}\right\}$. Since $\omega(G-X) \leq$ $|X|$, there exist $i, j, i \neq j$, such that $u_{i} w_{j} \in E(G)$. This is also true if $u_{l} w_{l} \notin E(G)$, otherwise $\omega\left(G-\left(X \cup\left\{u_{l}^{+}\right\}\right)\right)>\left|X \cup\left\{u_{l}^{+}\right\}\right|$. Choose $i$ minimal. By (OB1) and (OB2) we have $N\left(w_{i}\right) \subseteq\left(X-\left(\left\{y_{1}^{+}, y_{i}^{-}\right\} \cup\left\{y_{2}^{+}, y_{2}^{-}\right\}\right)\right) \cup\left\{u_{i}, u_{l}^{+}\right\}$, hence $d\left(w_{i}\right) \leq \bar{\delta}(G)-1$. Now consider the greatest $h$ such that $u_{g} w_{h} \in E(G)$ for some $g \neq h$, then we have $d\left(u_{h}\right) \leq$ $\bar{\delta}(G)-1$. Reasoning as in Case 1 we obtain a contradiction.

Case 3. $|Y|=3 \bar{\delta}(G)-n+|R|+2$.

By (4) and (8), $|X|=\bar{\delta}(G)$ and $|Z|=2$. So apart from the $X-1$ - and $X-2$-segments, $C$ contains either one $X-4$-segment, or two $X-3$-segments. Furthermore, by (OB3), $N\left(y_{0}\right)=N\left(y_{1}\right)=N\left(y_{2}\right)=N\left(y_{3}\right)=X$.

Case 3.1. $C$ contains a $X-4$-segment, say $u_{l} u_{l}^{+} w_{l}^{-} w_{l}$, and $u_{i} w_{j} \in E(G)$ for some $i, j, i \neq j$.

Choose $i$ minimal and $h$ maximal such that $u_{i} w_{j}, u_{g} w_{h} \in E(G)$ for some $j \neq i, g \neq h$. By (OB1) and (OB2) we have $i \leq g<h, N\left(w_{i}\right) \subseteq\left(X-\left(\left\{y_{1}^{+}, y_{1}^{-}\right\} \cup\left\{y_{2}^{+}, y_{2}^{-}\right\} \cup\left\{y_{3}^{+}, y_{3}^{-}\right\}\right)\right) \cup$ $\left\{u_{i}, u_{l}^{+}, w_{l}^{-}\right\}$and $N\left(u_{h}\right) \subseteq\left(X-\left(\left\{y_{1}^{+}, y_{1}^{-}\right\} \cup\left\{y_{2}^{+}, y_{2}^{-}\right\} \cup\left\{y_{3}^{+}, y_{3}^{-}\right\}\right)\right) \cup\left\{w_{h}, u_{l}^{+}, w_{l}^{-}\right\}$, so $d\left(w_{i}\right), d\left(u_{h}\right) \leq \bar{\delta}(G)-1$. Following Case 1 , we reach a contradiction.

Case 3.2. $C$ contains a $X-4$-segment, say $u_{l} u_{l}^{+} w_{l}^{-} w_{l}$, and $u_{i} w_{j} \notin E(G)$ for all $i, j, i \neq j$.

Since $G$ is 1 -tough, some $X-2$-segment contains a vertex adjacent to $u_{l}^{+}$or $w_{l}^{-}$. Without loss of generality, we may assume $w_{l}^{-} u_{i} \in E(G)$ or $w_{l}^{-} w_{i} \in E(G)$ for some $i \neq l$. First consider the case $w_{l}^{-} u_{i} \in E(G)$. By (lca), $w_{i} u_{l}^{+} \notin E(G)\left(u_{l}^{+} w_{i} u_{i} w_{l}^{-} \vec{C} u_{i}^{-} y_{0} w_{i}^{+} \vec{C} u_{l}^{+}\right)$.

Next we suppose $w_{l} u_{l}^{+} \in E(G)$. Then by (lca) we have $i<l\left(y_{1} \vec{C} u_{l}^{+} w_{l}\right.$ $\left.w_{i}^{-} u_{i} \vec{C} y_{1}^{-} y_{0} w_{l}^{+} \vec{C} u_{i}^{-} y_{1}\right)$ and $w_{i} w_{l}^{-} \notin E(G)\left(w_{i} w_{l}^{-} w_{l} u_{l}^{+} \vec{C} w_{i}^{+} y_{0} w_{l}^{+} \vec{C} w_{i}\right)$. Also, by (lca), $w_{i} y_{1}^{+} \notin E(G)\left(y_{1} w_{i}^{+} \vec{C} u_{l}^{+} w_{l} w_{l}^{-} u_{i} w_{i} y_{1}^{+} \vec{C} u_{i}^{-} y_{0} w_{l}^{+} \vec{C} y_{1}\right), w_{i} y_{1} \notin E(G)\left(y_{1}^{-} w_{i} u_{i} w_{l}^{-} w_{l} u_{l}^{+}{ }_{C}^{+} w_{i}^{+}\right.$ $\left.y_{1} \vec{C} u_{i}^{-} y_{0} w_{l}^{+} \vec{C} y_{1}^{-}\right)$. Similarly, we obtain $w_{i} y_{2}^{+}, w_{i} y_{2}^{-}, w_{i} y_{3}^{+}, w_{i} y_{3}^{-} \notin E(G)$. This gives 
$N\left(w_{i}\right) \subseteq\left(X-\left(\left\{y_{1}^{+}, y_{1}^{-}\right\} \cup\left\{y_{2}^{+}, y_{2}^{-}\right\} \cup\left\{y_{3}^{+}, y_{3}^{-}\right\}\right)\right) \cup\left\{u_{i}\right\}, \quad$ so $\quad d\left(w_{i}\right) \leq \bar{\delta}(G)-3$. We obtain the inequality

$$
\sigma_{3}(G) \leq d\left(w_{i}\right)+d\left(y_{0}\right)+d\left(y_{1}\right) \leq 3 \bar{\delta}(G)-3 .
$$

This contradiction shows that $w_{l} u_{l}^{+} \notin E(G)$. Also, by (lca), $w_{l} u_{l} \notin E(G)$ $\left(u_{i} \vec{C} u_{l}^{-} y_{0} u_{i}^{-} \overleftarrow{C} w_{l} u_{l} u_{l}^{+} w_{l}^{-} u_{i}\right)$. This means $N\left(w_{l}\right) \subseteq X \cup\left\{w_{l}^{-}\right\}$and $X^{+} \cup\left\{w_{l}\right\}$ is an independent set. So, by (6), (7), and (10), $R \cup Y \cup\left\{w_{l}\right\}$ is a suitable set.

If $w_{l}^{-} w_{i} \in E(G)$, then similar conclusions are obtained by considering $u_{i}$ instead of $w_{i}$.

Case 3.3. $C$ contains two $X-3$-segments, say $u_{l} u_{l}^{+} w_{l}$ and $u_{m} u_{m}^{+} w_{m}(l<m)$.

Let $k \in\{l, m\}$. If $N\left(u_{k}^{+}\right) \cap(U \cup W) \neq\left\{u_{k}, w_{k}\right\}$, then we have, by (lca), $u_{k} w_{k} \notin E(G)$ $\left(u_{i} \vec{C} u_{k}^{-} y_{0} u_{i}^{-} \stackrel{\leftarrow}{C} w_{k} u_{k} u_{k}^{+} u_{i}\right.$, if $u_{k}^{+} u_{i} \in E(G)$ for some $i \neq k ; w_{i} u_{k}^{+} w_{k} u_{k} \stackrel{\leftarrow}{C} w_{i}^{+} y_{0} w_{k}^{+} \vec{C} w_{i}$, if $u_{k}^{+} w_{i} \in E(G)$ for some $\left.i \neq k\right)$. Since $G$ is 1-tough, $\omega(G-T) \leq|T|$ for $T=X, X \cup$ $\left\{u_{l}^{+}\right\}, X \cup\left\{u_{m}^{+}\right\}, X \cup\left\{u_{l}^{+}, u_{m}^{+}\right\}$. It follows that $u_{i} w_{j} \in E(G)$ for some $i, j, i \neq j$. Choose $i$ minimal and $h$ maximal such that $u_{i} w_{j}, u_{g} w_{h} \in E(G)$ for some $j \neq i, g \neq h$. By (OBI) and (OB2), $i \leq g<h, N\left(w_{i}\right) \subseteq\left(X-\left(\left\{y_{1}^{+}, y_{1}^{-}\right\} \cup\left\{y_{2}^{+}, y_{2}^{-}\right\} \cup\left\{y_{3}^{+}, y_{3}^{-}\right\}\right)\right) \cup\left\{u_{i}, u_{1}^{+}, u_{m}^{+}\right\}$ and $N\left(u_{h}\right) \subseteq\left(X-\left(\left\{y_{1}^{+}, y_{1}^{-}\right\} \cup\left\{y_{2}^{+}, y_{2}^{-}\right\} \cup\left\{y_{3}^{+}, y_{3}^{-}\right\}\right)\right) \cup\left\{w_{h}, u_{l}^{+}, u_{m}^{+}\right\}$. As in Case 3.1, we obtain a contradiction.

Case 4. $|Y| \geq 3 \bar{\delta}(G)-n+|R|+3$.

We have $|Y \cup R| \geq 3 \bar{\delta}(G)-n+2|R|+3$, so by (6), (7), and (10), $R \cup Y$ is a suitable set.

Proof of Theorem 7. Suppose first that $|Y \cup R| \geq t$. Set $S=Y \cup R$. By Lemma 18 (a) and (6) we obtain

$$
|V(C)| \geq|X|+\left|X^{+}\right|=2|X| \geq 2|N(S)| \geq 2 \mathrm{NC}_{|S|}(G) \geq 2 \mathrm{NC}_{t}(G)
$$

and hence we are done.

So from now on we may assume $|Y \cup R| \leq t-1$. Hence (5) gives

$$
t \geq|Y|+|R|+1 \geq 3 \bar{\delta}(G)-n+2|R|+1 .
$$

If $c(G) \geq \frac{1}{3}(2 n+4 \bar{\delta}(G)+1-2 t)$, then we are also done. Hence we may assume $c(G) \leq \frac{1}{3}(2 n+4 \bar{\delta}(G)-2 t)$. Together with $-\frac{1}{2} n \leq \frac{1}{2} t-\frac{3}{2} \bar{\delta}(G)-|R|-\frac{1}{2}$, by (11), this gives

$$
t \leq n+2 \bar{\delta}(G)-\frac{3}{2}(n-|R|) \leq \frac{1}{2} t+\frac{1}{2} \bar{\delta}(G)+\frac{1}{2}|R|-\frac{1}{2} .
$$

This is equivalent to $t+1 \leq \bar{\delta}(G)+|R|$, and so (4) gives

$$
|Y|+|W|+|R|=|X|+|R| \geq \bar{\delta}(G)+|R| \geq t+1
$$

So we can choose $Q_{1}=y_{1} \vec{C} w_{q}$ with $w_{q} \in W$ and $\left|W \cap Q_{1}\right|+|Y|+|R|=t$. Note that by this definition

$$
\left|U \cap Q_{1}\right|=\left|W \cap Q_{1}\right|=t-|Y|-|R|
$$


Let $T=\left(W \cap Q_{1}\right) \cup Y \cup R$. Then, by Lemma 18, Corollary 21 (a) and the definitions of $X$ and $W, T$ is an independent set of $t$ vertices with $N(T) \subseteq V(C)-X^{-}$. If $|N(T)| \leq \frac{1}{2}|V(C)|$, then we are done. So we assume

$$
|N(T)| \geq \frac{1}{2}(|V(C)|+1)=\frac{1}{2}(n-|R|+1) .
$$

We will show that this assumption eventually leads to a contradiction.

Set $Q_{2}=V(C)-Q_{1}$ and note that

$$
\begin{aligned}
Q_{2}-(X \cup Z) & =V(C)-\left(Q_{1} \cup X \cup\left(Z \cap Q_{2}\right)\right) \\
& =V(C)-\left(\left(Q_{1}-X\right) \cup X \cup\left(Z \cap Q_{2}\right)\right)
\end{aligned}
$$

Let $J=N(T) \cap\left(Q_{2}-(X \cup Z)\right)$. Since $N(T) \subseteq V(C)$, we have

$$
|J|=|N(T)|-\left|N(T) \cap\left(Q_{1}-X\right)\right|-|N(T) \cap X|-\left|N(T) \cap\left(Z \cap Q_{2}\right)\right| .
$$

Clearly,

$$
\begin{aligned}
|N(T) \cap X| & \leq|X| \\
\left|N(T) \cap\left(Z \cap Q_{2}\right)\right| & \leq\left|Z \cap Q_{2}\right|,
\end{aligned}
$$

and, since $N(T) \subseteq V(C)-\left(X^{-} \cup Y\right)$,

$$
\left|N(T) \cap\left(Q_{1}-X\right)\right| \leq\left|U \cap Q_{1}\right|+\left|Z \cap Q_{1}\right| .
$$

Using (8) we obtain

$$
|Y|=|Z|+3|X|-n+|R|
$$

Combing (13)-(19) gives

$$
\begin{aligned}
|J| & \geq|N(T)|-\left|U \cap Q_{1}\right|-\left|Z \cap Q_{1}\right|-|X|-\left|Z \cap Q_{2}\right| \\
& \geq \frac{1}{2}(n-|R|+1)-t+|Y|+|R|-|X|-|Z| \\
& =\frac{1}{2}(n-|R|+1)-t+|Z|+3|X|-n+|R|+|R|-|X|-|Z| \\
& =2|X|+\frac{3}{2}|R|-\frac{1}{2} n-t+\frac{1}{2} .
\end{aligned}
$$

Let $K=N\left(y_{0}\right) \cap N\left(y_{1}\right)$. Since $N\left(y_{0}\right), N\left(y_{1}\right) \subseteq X$ and $d\left(y_{0}\right), d\left(y_{1}\right) \geq \bar{\delta}(G)$, we have

$$
|K|=\left|N\left(y_{0}\right)\right|+\left|N\left(y_{1}\right)\right|-\left|N\left(y_{0}\right) \cup N\left(y_{1}\right)\right| \geq 2 \bar{\delta}(G)-|X| .
$$

Combining (20) and (21) gives, using (12),

$$
\begin{aligned}
\left|J^{-} \cap K\right|+\left|J^{-} \cup K\right| & =\left|J^{-}\right|+|K|=|J|+|K| \\
& \geq 2|X|+\frac{3}{2}|R|-\frac{1}{2} n-t+\frac{1}{2}+2 \bar{\delta}(G)-|X| \\
& \geq|X|+\frac{3}{2}|R|-\frac{1}{2} n-2 \bar{\delta}(G)-\frac{3}{2}|R|+\frac{1}{2} n+\frac{1}{2}+2 \bar{\delta}(G) \\
& =|X|+\frac{1}{2} .
\end{aligned}
$$


Since $N(T) \subseteq V(C)-X^{-}$, it follows that $J \subseteq X^{+}$. Therefore we have $J^{-} \cup K \subseteq X$. Hence, by (22) there exists a vertex $x \in J^{-} \cap K$. Since $K \subseteq X$, we have $x \in X$. Let $w \in T$ such that $w x^{+} \in E(G)$, then we have $w \in W \cap Q_{1}$, since $N(Y \cup R) \subseteq X$. Let $P$ be the path $w^{+} \vec{C} x y_{1} \vec{C} w x^{+} \vec{C} y_{1}^{-}$. Then $P$ is a hopping path, as defined in Section 3. Conditions (HP1) and (HP2) are obvious and the only vertex in $Y$ for which (HP3) is not obvious, is $y_{1}$. But the neighbors of $y_{1}$ on $P$ are $x$ and $y_{1}^{+}$which are both neighbors of $y_{0}$, hence are elements of $X_{1}$. By Lemma 20 , the existence of the path $P$ gives us the promised contradiction.

\section{ACKNOWLEDGMENT}

The author likes to thank the anonymous referees for their very helpful and stimulating suggestions which greatly improved the presentation of the paper.

\section{References}

[1] D. Bauer, H. J. Broersma, and H. J. Veldman, Around three lemmas in hamiltonian graph theory, in Topics in Combinatorics and Graph Theory, Essays in Honour of Gerhard Ringel, R. Bodendiek and R. Henn, eds. Physica-Verlag, Heidelberg (1990), $101-110$.

[2] D. Bauer, A. Morgana, E. F. Schmeichel, and H. J. Veldman, Long cycles in graphs with large degree sums, Discrete Math. 79 (1989/90), 59-70.

[3] D. Bauer, E. Schmeichel, and H. J. Veldman, Some recent results on long cycles in tough graphs, in Graph Theory, Combinatorics, and Applications. Proceedings of the Sixth Quadrennial International Conference on the Theory and Applications of Graphs, Western Michigan University, Y. Alavi, G. Chartrand, O. R. Oellerman, and A. J. Schwenk, eds. John Wiley \& Sons, New York (1991), 113-123.

[4] J. A. Bondy, Longest paths and cycles in graphs of high degree. Research Report CORR 80-16, Univ. of Waterloo, Waterloo, Ontario (1980).

[5] J. A. Bondy and U. S. R. Murty, Graph Theory with Applications, Macmillan, London and Elsevier, New York (1976).

[6] H. J. Broersma, J. van den Heuvel, and H. J. Veldman, Long cycles, degree sums and neighborhood unions. Discrete Math. 121 (1993), 25-35.

[7] G. A. Dirac, Some theorems on abstract graphs. Proc. London Math. Soc. (3) 2 (1952), 69-81.

[8] H. Enomoto, J. van den Heuvel, A. Kaneko, and A. Saito, Relative length of long paths and cycles in graphs with large degree sums. Preprint (1993).

[9] R. Häggkvist, On the structure of non-hamiltonian graphs I. Combinatorics, Probability and Computing 1 (1992), 27-34.

[10] B. Jackson, Hamilton cycles in regular 2-connected graphs. J. Combinatorial Theory (B) 29 (1980), 27-46.

[11] H. Li, Circumferences in 1-tough graphs. Preprint (1993).

[12] Min Aung, Circumference of a regular graph. J. Graph Theory 13 (1989), 149-155.

[13] C.St. J.A. Nash-Williams, Edge-disjoint hamiltonian circuits in graphs with vertices of large valency, in Studies in Pure Mathematics. ed. L. Mirsky. Academic Press, London (1971), 157-183.

[14] D. R. Woodall, The binding number of a graph and its Anderson number. $J$. Combinatorial Theory (B) 15 (1973), 225-255.

Received June 10, 1994 